\title{
After years of neglect, Brazil takes aim at Chagas disease
}

In the 1990s, Brazil made headlines for cutting HIV infection rates to well below projected levels, but the country has not mobilized itself as energetically against other public health challenge since then. Chagas disease, a parasitic infection first described over a century ago by the Brazilian physician Carlos Chagas, is carried by 3 million Brazilians-more than are now afflicted by HIV-with close to 5,000 of those people dying from the disease each year. Yet Chagas has remained sorely neglected, and, to this day, most people with the disease are treated with decades-old drugs that fail to completely eliminate the Trypanosoma cruzi protozoan responsible for the chronic condition.

The sluggish pace of development for Chagas treatments stems in part from the relative lack of support from public health authorities. Unlike HIV, for which Brazil's Ministry of Health eventually leapt into action, progress for combating Chagas has been far more gradual, largely falling on the research community, which has pushed for government programs. Five years ago, for example, parasite transmission by the Southern Cone's most common Chagas vector, the blood-sucking triatoma bug, was effectively halted in Brazil through a sustained national campaign of insecticide spraying and housing improvement projects. And research into innovative therapies is starting to bear fruit.

"The intense effort of the scientific Brazilian community called attention to a large health problem and generated epidemiological and clinical data," says Tania Araújo-Jorge, director of the Oswaldo Cruz Institute in Rio de Janeiro.

For instance, Brazil is now home to two ongoing phase 3 trials for Chagas drugs: the so-called BENEFIT trial, a study involving more than 1,000 participants that aims to determine whether benznidazole, one of the two currently used antiparasitic drugs for the disease, helps people with chronic as well as the more serious, acute form of Chagas; and a smaller, 130-person trial testing whether the micronutrient selenium prevents the heart problems associated with chronic infection. Both are due to finish in 2013. (Elsewhere, a small crop of earlier-stage trials for new compounds against Chagas have been launched in the US, Spain, Argentina and Bolivia.)

In addition, attention is now finally being paid to the problem of HIV-Chagas co-infection. In 2006, a network of Brazilian scientists was set up to find answers to basic research questions such as how many people with Chagas also carry HIV. And, in a paper published in August, a team led by Maria Shikanai-Yasuda, an infectious disease researcher at the University of São Paulo, reported that these co-infected individuals tend to have T. cruzi levels that are several orders of magnitude greater than those who are HIV negative (PLoS Negl. Trop. Dis. 5, e1277, 2011). This suggests that co-infected people may particularly benefit from taking benznidazole to keep their parasite loads in check - and, as such, provides some hope where there was little before.

Anna Petherick

\section{Hopes build that new infrastructure can aid drug discovery}

RIO DE JANEIRO - Four years ago, Carlos Morel, a biophysicist at the Oswaldo Cruz Foundation (Fiocruz), penned an opinion piece urging Brazil to improve its research infrastructure to address the country's public health challenges (Nature 449, 180-182, 2007). Now that call to action is close to a reality, thanks to the nearly complete, five-story Center for Technological Development in Health (CDTS), which is slated to open here on the Fiocruz campus next year. "This building represents a dream come true for the entire Fiocruz community," says Morel, beaming like an expectant father.

Fiocruz is one of the most prominent biomedical research institutions in all of Latin America. Like a geographically dispersed version of the US National Institutes of Health, the public organization employs thousands of scientists at 15 units throughout Brazil. Fiocruz is also a major producer of therapeutics, capable of delivering 2 billion drug units and 200 million doses of vaccines every year. Nevertheless, it suffers from a problem common to Brazilian science as a whole: it fails to convert the

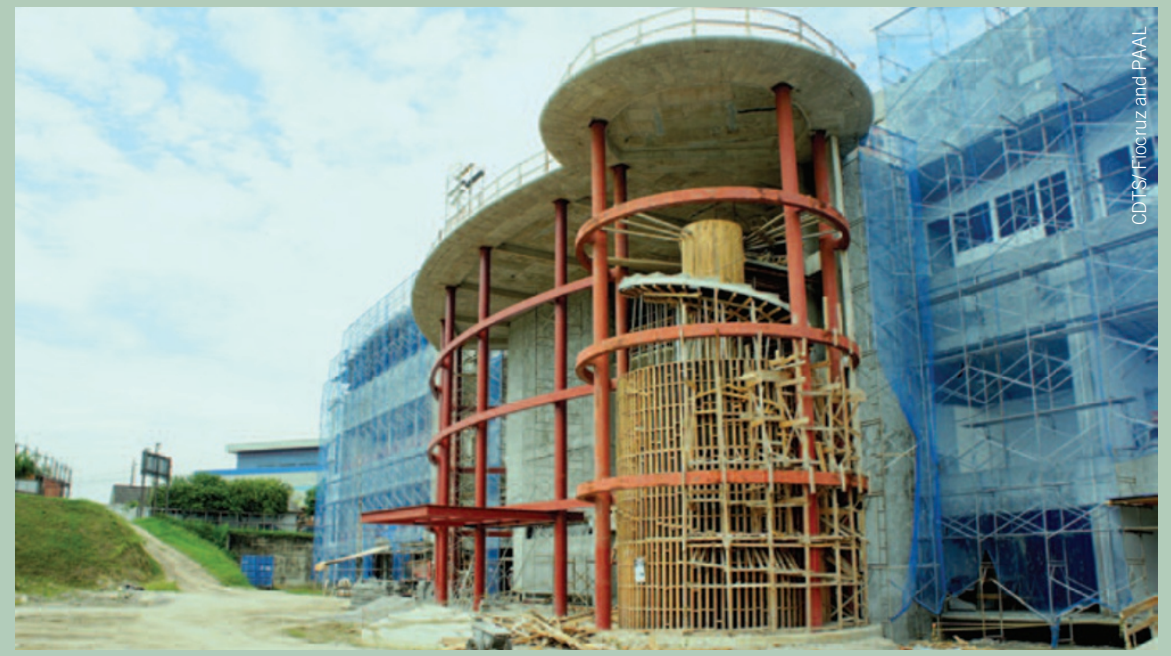

Spiraling up: Fiocruz's near-finished building aims to boost translational research.

knowledge gleaned by its researchers into

biotech solutions.

"Fiocruz researchers publish over 1,600 papers in indexed journals every year," says Morel, director of the CDTS. "Yet, the institution owns only a hundred patents."

Morel and his colleagues-not to mention his financial backers-hope this building can help foster translational innovation and drive commercial success. When it opens next fall, the CDTS, built with a 140-million-Brazilian-reais (\$84 million) investment from the federal government, will be equipped with state-of-the-art molecular biology labs for basic research, a high-throughput screening core for biotech prospection and animal facilities for preclinical proof-of-concept studies. 